\title{
La nutrition de la personne âgée : une des pierres angulaires du parcours de santé des personnes âgées en risque de perte d'autonomie
}

\author{
Nutrition in the elderly: one of the cornerstones of the healthcare pathway for elderly patients at \\ risk of losing their independence
}

\author{
C. Jeandel \\ (C) Springer-Verlag France 2013
}

Les prévisions démographiques portant sur les pays développés font état pour l'horizon 2050 d'un doublement de la population âgée de 60 ans, d'un triplement des 75 ans ou plus et d'une multiplication par un facteur 5 de la population âgée de plus de 85 ans.

Avec l'avancée en âge, le nombre moyen d'affections déclarées augmente. Ainsi, au-delà de 64 ans, sept affections sont en moyenne déclarées dont certaines sont des maladies chroniques à l'origine d'une dépendance physique et/ou psychique.

Les données de la CNAMTS révèlent que : $85 \%$ des personnes de 75 ans et plus présentent au moins une pathologie (une pathologie cardiovasculaire pour $29 \%$ d'entre elles), que $33 \%$ ont été hospitalisées au moins une fois en 2010 (dont au moins une fois en urgence pour $44 \%$ d'entre elles), pour une durée de 12 jours en moyenne, qu'en moyenne, sept molécules différentes leur sont délivrées au moins trois fois par an et enfin que $57 \%$ présentent une affection de longue durée (ALD).

Quatre facteurs majeurs d'hospitalisation de ces populations ont par ailleurs été ciblés : la iatrogénie médicamenteuse en premier lieu (mais aussi difficultés d'observance et insuffisances de traitement), la dénutrition, la dépression et les chutes.

Face à ces enjeux démographiques, épidémiologiques, sanitaires et à leurs impacts financiers, nos pouvoirs publics sont très largement convaincus de la nécessaire révision de nos modèles d'organisation tels qu'ils ont été proposés à travers différents rapports récents (Haut Conseil pour l'avenir de l'assurance maladie [HCAAM], personnes âgées en risque de perte d'autonomie [Paerpa]) [1].

\footnotetext{
C. Jeandel $(\bowtie)$

Service de gérontologie clinique, centre de gérontologie clinique Antonin-Balmes, CHU de Montpellier, 39, avenue CharlesFlahaut, F-34295 Montpellier cedex 05, France e-mail : claudejeandel@yahoo.fr
}

Ainsi, le HCAAM a énoncé le principe qu'une amélioration durable de la coordination entre les acteurs du système de santé devait permettre d'optimiser la prise en charge des Paerpa et s'accompagner d'une source d'économies pour l'assurance maladie. Un Comité national de pilotage (Copil) a été installé en avril 2012 pour instruire une large concertation autour de cet enjeu en prenant en compte la coopération et la coordination des acteurs en ville, en établissement de santé et en EHPAD, le développement professionnel continu (DPC) et les systèmes d'information. Les conclusions ont fait l'objet d'un rapport de mission et d'un cahier des charges remis au ministère dans l'optique de l'expérimentation de prototypes de " parcours » des Paerpa devant débuter en septembre 2013 sur les territoires retenus.

L'ensemble des réflexions convergent sur le fait que le modèle de Parcours de Santé s'impose désormais comme un nouveau paradigme structurant de l'action sanitaire et sociale et des programmes régionaux de santé (PRS) des Agences régionales de santé (ARS).

Centré sur les besoins et promouvant l'approche personnalisée, ce modèle doit concourir à faire en sorte qu'une population reçoive les bons soins par les bons professionnels dans les bonnes structures au bon moment et au meilleur coût.

Ce modèle a donc pour finalité d'accroître la pertinence et la qualité des soins et des aides dont bénéficient les personnes âgées, et ainsi d'améliorer, sur un plan individuel, leur qualité de vie et celle de leurs aidants, et sur un plan collectif, l'efficience de leur accompagnement. La notion de Paerpa concerne l'ensemble des personnes de 75 ans et plus en situation de fragilité ou encore autonomes mais dont l'état de santé est susceptible de se dégrader pour des raisons médicales et/ou sociales. Les acteurs qui en assurent la prise en charge sont multiples et leurs actions insuffisamment coordonnées. La conduite des travaux a été centrée sur le modèle économique suivant : un moindre recours à l'hospitalisation traditionnelle en situation de crise (non programmée) afin de renforcer les moyens dédiés aux équipes de 
proximité, aux consultations et hôpitaux de jour gériatriques, à l'expertise gériatrique en ville, à la télémédecine...

En ville, l'objectif est de formaliser la mobilisation des professionnels de proximité au plus près des personnes âgées et au plus tôt dans le parcours. À cette fin, il est préconisé le développement d'une coordination clinique de proximité rassemblant, autour du médecin traitant, un infirmier, un pharmacien d'officine et éventuellement un masseurkinésithérapeute. Chacun de ces acteurs doit se recentrer sur le plus haut niveau de ses compétences. Ce travail passera par deux outils principaux : une formation spécifique et l'élaboration d'un plan personnalisé de santé (PPS).

Afin de faciliter le recours des équipes de proximité, le comité préconise un rapprochement des différents dispositifs de coordination et d'intégration dans le cadre d'une coordination territoriale d'appui sur un territoire plus large que la coordination clinique de proximité.

Les établissements de santé devront quant à eux mettre en œuvre les mesures contribuant à améliorer les transitions ville-hôpital et à réduire le taux de réhospitalisations précoces telles que le repérage précoce des personnes âgées fragiles ou en risque de perte d'autonomie, l'évaluation précise de leurs besoins médicopsychosociaux afin d'adapter les réponses dans le cadre d'un PPS, la prévention des risques induits par l'hospitalisation pouvant se référer à différents référentiels de pratiques, l'anticipation des modalités de sortie et l'optimisation des échanges d'informations (compte rendu d'hospitalisation) permettant le retour à domicile dans les conditions les mieux adaptées. Ils devront également adapter leur offre afin de permettre aux acteurs de la ville et aux EHPAD de bénéficier de leur expertise (hospitalisation de jour et plateforme de fragilité, filière gériatrique, expertise gérontopsychiatrique et en soins palliatifs, hospitalisation à domicile). Pour ce faire, ils pourront s'appuyer sur une " commission de coordination gériatrique " sur le modèle des sous-commissions de la CME existantes.

L'amélioration du parcours nécessite également la mise en place d'un système d'information partagé par les acteurs des trois secteurs : annuaire des ressources sanitaires, médicosociales et sociales et messagerie sécurisée.

Ce numéro thématique portant sur la dénutrition du sujet âgé, sur ses conséquences et sa prise en charge s'avère particulièrement adapté aux enjeux de la médecine de parcours qui doit s'appuyer sur le déploiement des bonnes pratiques en priorisant notamment la prévention des accidents médicamenteux, la prévention des chutes, et la prévention et prise en charge de la dénutrition du sujet âgé.

\section{Référence}

1. Libault D (2013) Parcours de santé des personnes âgées en risque de perte d'autonomie. Rapport sur la mise en œuvre des projets pilotes 